\title{
Quantitative Analysis of the RPC Vessel Density and the RNFL Thickness in Patients with Type 2 Diabetes Mellitus by Using OCT Angiography
}

\author{
Min Zhang ${ }^{a} \quad$ Fan Jia $^{a} \quad \mathrm{Na} \mathrm{Li}^{\mathrm{b}} \quad$ Chunyuan Song $^{\mathrm{a}} \quad$ Jin Yang $^{\mathrm{a}}$ Kaili Yang $^{\mathrm{b}}$ \\ Yan Li ${ }^{\text {b }}$ Shuna Wang ${ }^{\mathrm{b}}$

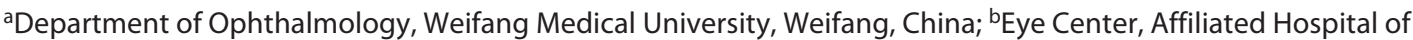 \\ Weifang Medical University, Weifang, China
}

\section{Keywords}

Diabetic retinopathy - Optical coherence tomography angiography · Optic disc · Radial peripapillary capillaries .

Vessel density $\cdot$ Retinal nerve fibre layer

\begin{abstract}
Introduction: This study aims to compare the structural differences in the optic disc blood perfusion and the peripapillary retinal nerve fibre layer ( $p R N F L$ ) thickness in agematched healthy subjects and patients with type 2 diabetes mellitus (DM) by using quantitative analysis with optical coherence tomography angiography (OCTA). Methods: A cross-sectional cohort study on patients with type $2 \mathrm{DM}$ with or without diabetic retinopathy (DR) and healthy subjects was conducted. The 4.5-mm scanning angio-disc pattern of the OCTA system was used to assess the optic disc. The analysed indices included radial peripapillary capillary (RPC) vessel density and pRNFL thickness. Results: A total of 78 eyes from 78 patients with type $2 \mathrm{DM}$, including 27 without clinical DR (NDR), 26 with non-proliferative DR (NPDR), and 25 with proliferative DR (PDR), and 28 age-matched healthy subjects were enrolled. The average RPC vessel density of the whole $(p<0.001)$ and the peripapillary $(p<0.001)$ regions was significantly different in different groups, whereas the pRNFL was not statistically significant ( $p=0.764)$. Compared with that in healthy subjects, the RPC vessel densities in 4,5 , and 8 peripapillary sectors in NDR (all $p<0.05$ ), NPDR
\end{abstract}

karger@karger.com www.karger.com/ore

Karger $\stackrel{\text { ' }}{5}$
(C) 2021 The Author(s)

Published by S. Karger AG, Basel

This is an Open Access article licensed under the Creative Commons Attribution-NonCommercial-4.0 International License (CC BY-NC) (http://www.karger.com/Services/OpenAccessLicense), applicable to the online version of the article only. Usage and distribution for commercial purposes requires written permission. (all $p<0.05$ ), and PDR (all $p<0.05$ ) groups, respectively, were reduced. Compared with that in healthy subjects, the pRNFL thickness significantly decreased in the inferior nasal sector $(p=0.001)$ in NDR but significantly increased in the 2 sectors (all $p<0.01$ ) in PDR. The DR severity was negatively correlated with the peripapillary RPC vessel density $(r=-0.583$, $p<0.001$ ) but had no correlation with the PRNFL thickness $(r=-0.045, p=0.648)$. The positive correlation between the peripapillary RPC vessel density and the pRNFL thickness was statistically significant in the control $(r=0.531, p=0.004)$, $\operatorname{NDR}(r=0.528, p=0.004)$, and NPDR $(r=0.405, p=0.040)$ groups but not in the PDR group ( $r=0.394, p=0.05)$. Conclusions: The peripapillary RPC perfusion decreased with DR aggravation, which may be considered as a useful indicator of DR severity. However, the pRNFL thickness had little diagnostic power in differentiating healthy and DM eyes.

\footnotetext{
(c) 2021 The Author(s)

Published by S. Karger AG, Basel
}

\section{Introduction}

The prevalence of diabetes mellitus (DM) continues to rise rapidly and globally. The data from the International Diabetes Federation predict that 578 million people worldwide will have DM over the next decade $[1,2]$. As one of the common chronic microvascular complications, diabetic retinopathy (DR) occurs in more than one-third of patients with DM and is the leading cause of blindness in the elder-
Correspondence to:

Yan Li, liyanmails@126.com

Shuna Wang, wangshuna80@126.com 
ly [3-5]. The pathophysiology of DR generally results from the thickening of the basement membrane, pericyte loss, and endothelial cell apoptosis, which may lead to microaneurysm formation and capillary occlusion and increase the vascular permeability by some ischaemia-activated growth factors $[6,7]$. Mitochondrial damage and oxidative stress are associated with retinal neurodegeneration $[8,9]$. DR is a microvascular disease and a neurodegenerative disease [10]. Many studies indicate that neuroretinal structural changes appear before evident vascular involvement [11]. Improvements in predicting the occurrence and monitoring the progress of DR are urgently needed due to insidious onset [12-14]. Fluorescein angiography enables the visualization of retinal circulation but has potential risks of intravenous contrast agents and difficulty in imaging the radial peripapillary capillary (RPC) region [15-17]. The optical coherence tomography angiography (OCTA) quantitatively detects perfusion volume through the high-resolution identification of moving red blood cells. The early identification of microcirculation disturbance and neurodegeneration by using OCTA is important. Most studies on DR have paid attention to the macular area in the past, whereas the focus on the optic disc is limited [18-20]. Interestingly, the axon density in the optic papilla is higher than that in the macular [21]. Thus, the retinal nerve fibre layer (RNFL) of the optic disc may be important in studying the neurodegeneration of DR. RPC is clinically and histologically confirmed to play a critically pivotal role in the arcuate fibres of RNFL [22, 23]. Therefore, the RPC vessel density in the optic disc may be a good assessment index. Previous studies reveal some quantitative indicators to describe the gradual changes in the peripapillary RPC vessel density and the pRNFL thickness [17, 24, 25]. However, few studies have included 8 sections of the peripapillary RPC vessel density in patients with DR at all stages. The optic nerve head can be comprehensively evaluated by analysing the peripapillary RPC vessel density and the pRNFL thickness in 8 sections. Similarly, by including all stages of DR, the development of DR can be fully clarified. The OCTA is used to quantitatively detect the structural changes in the RPC vessel density and the pRNFL thickness of the optic disc in patients with DM and evaluate its use in predicting the occurrence of DR.

\section{Materials and Methods}

\section{Subjects}

This prospective cross-sectional study adhered to the guidelines of the Declaration of Helsinki and was approved by the Research Ethics Committee of the Affiliated Hospital of Weifang Medical
University (Approval No. 2019-010). The subjects were recruited from our department between March 2019 and January 2020, and subjects who met the criteria were consecutively enrolled.

Informed consent was obtained from all subjects to be included in our study. Healthy persons undergoing physical examinations were chosen as the control group. From these subjects, only those who met the inclusion (except DM diagnosis) and the exclusion criteria were included.

The inclusion criteria were as follows: diagnosis of type $2 \mathrm{DM}$, clear refractive media, ametropia $<3$ dioptres, and intraocular pressure $\leq 21 \mathrm{~mm} \mathrm{Hg}$. The exclusion criteria were as follows: presence of abnormal ocular circulation disease (such as glaucoma, optic neuropathy, macular oedema, and retinal vessel occlusion), history of any treatment for DR, hypertension, allergic constitution, or cardio-cerebrovascular disease.

\section{Ophthalmic Examination}

Each subject underwent comprehensive ophthalmic examinations, which included intraocular pressure, slit lamp microscopy, and ophthalmoscopy. Furthermore, the same senior ophthalmologist operated the colour fundus photography (Cidnary Medical Ltd., Wuhan, China) and OCTA (Optovue Inc., Fremont, CA, USA) after mydriasis. The eyes of the patients were divided into 3 groups, in accordance with the International Clinical Diabetic Retinopathy Disease Severity Scale [26], based on mydriatic fundus examination: (1) no abnormalities (DM with no DR, NDR); (2) microaneurysms, intraretinal haemorrhages, venous beading, intraretinal microvascular abnormalities, soft exudates, or hard exudates (non-proliferative DR, NPDR); and (3) neovascularization and vitreous/preretinal haemorrhage (proliferative DR, PDR). If both eyes were at the same stage, the severe eye was selected.

\section{OCTA Data Measurements}

The angio-disc 4.5-mm scan mode of the OCTA device with an $840-\mathrm{nm}$ light source was chosen to capture a $4.5 \mathrm{~mm} \times 4.5 \mathrm{~mm}$ area centred on the optic disc. The average vessel density over the entire range of measurements is defined as the whole VD. The software automatically obtained and segmented the optic disc images through an automatic fitting scan. The scope of the optic disc is automatically determined by the system, and the range within the boundary of the optic disc is defined as an inside disc. The peripapillary region was defined as a $750-\mu \mathrm{m}$ wide annulus extending from the optic disc boundary and divided into 8 sectors, namely, nasal superior (NS), nasal inferior (NI), inferior nasal (IN), inferior tempo (IT), tempo superior (TS), tempo inferior (TI), superior nasal (SN), and superior tempo (ST). The RPC vascular density and the NFL thickness of each segment were analysed. Each eye was measured thrice. Images with scanning quality $<5$ were excluded, and the highest-quality image of the subject was selected for preservation.

\section{Statistical Analysis}

Multivariate statistical analysis was completed using SPSS 25.0 (IBM Corp., Armonk, NY, USA). The qualitative data of gender and laterality were expressed using frequency, and the $\chi^{2}$ test was used to evaluate significant differences between groups. The Shapiro-Wilk test was used to evaluate the normality of quantitative data distribution. The quantitative data were presented as mean values \pm standard deviation or median (interquartile range). The differences between groups were evaluated using analysis of variance and the Kruskal-Wallis test with Bonferroni correction. The 
Table 1. Clinical and demographic characteristics of the study population

\begin{tabular}{llllll}
\hline & Control & NDR & NPDR & PDR & $p$ value \\
\hline Patients, $n$ & 28 & 27 & 26 & 25 & NA \\
Age, mean \pm SD, years & $52.93 \pm 13.32$ & $47.93 \pm 13.50$ & $53.92 \pm 9.83$ & $54.40 \pm 7.08$ & $F=1.624, p=0.194$ \\
Gender, male/female & $14 / 14$ & $11 / 16$ & $18 / 8$ & $13 / 12$ & $\chi^{2}=4.487, p=0.213$ \\
Laterality, right/left & $15 / 13$ & $10 / 17$ & $14 / 12$ & $14 / 11$ & $\chi^{2}=2.472, p=0.480$ \\
DM duration, median (IQR), years & - & $2.0(0.3,8.0)$ & $8.5(3.0,15.5)$ & $10.0(7.0,12.5)$ & $H=69.972, p<0.001^{*}$ \\
\hline
\end{tabular}

NA, not applicable; $\chi^{2}, \chi^{2}$ test; $F$, Welch test; $H$, Dunnett's $t$ test; DM, diabetes mellitus. * Statistical differences.

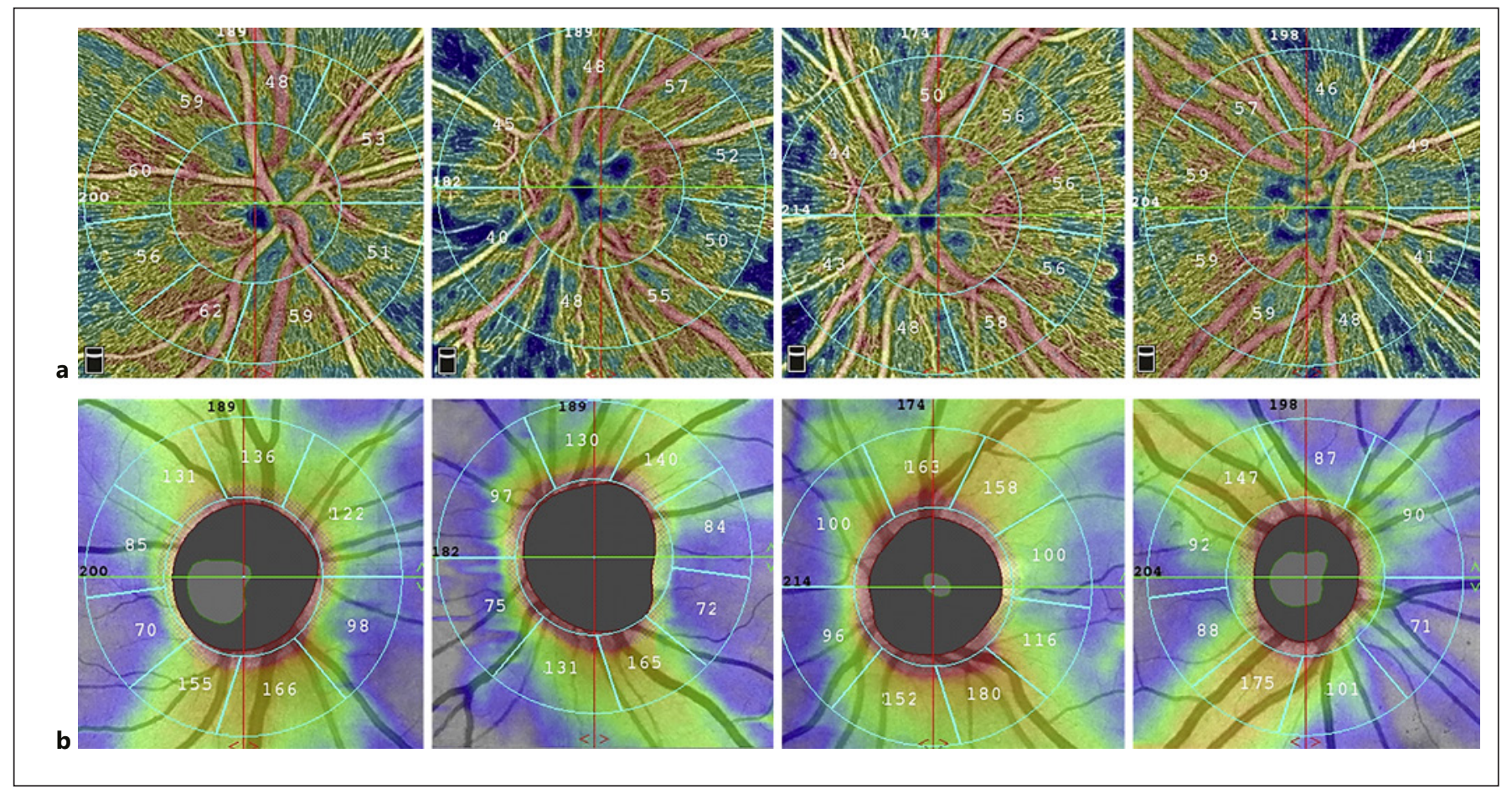

Fig. 1. Representative OCTA images of samples showing the vessel densities and the pRNFL thickness in the optic disc of each group (control, NDR, NPDR, and PDR). Optic disc neurovascular map measured through a 4.5 $\mathrm{mm} \times 4.5 \mathrm{~mm}$ scan by using OCTA. a Radial peripapillary capillary layer is a $750-\mu \mathrm{m}$-wide annulus extending from the optic disc boundary and divided into 8 sectors. $\mathbf{b}$ En face OCTA of the RNFL thickness in the peripapillary region. OCTA, optical coherence tomography angiography; RNFL, retinal nerve fibre layer.

correlation coefficient was analysed using the Spearman's rank correlation. Results with a $p$ value $<0.05$ were considered statistically significant.

\section{Results}

\section{Population Characteristics}

A total of 28 eyes from 28 control individuals and 78 eyes from patients with type $2 \mathrm{DM}$ were eligible for the study. The 78 eyes of the patients with DM were divided into 3 groups on the basis of the DR staging. The NDR, NPDR, and PDR groups contained 27, 26, and 25 eyes. As shown in Table 1, no statistical difference was observed in age, gender, and laterality within each group $(p>0.05)$. The duration of DM among the 3 DM groups was comparable $(p<0.001)$.

\section{OCTA Measurements of the Optic Disc}

Figure 1 shows the representative OCTA image samples of the vessel densities and the pRNFL thickness in the optic disc. The RPC vessel density in the whole region, 
Table 2. Representative RPC vessel density and RNFL thickness for the normal and diabetic groups

\begin{tabular}{|c|c|c|c|c|c|c|}
\hline \multicolumn{7}{|l|}{$\mathrm{RPC}$, mean $\pm \mathrm{SD}, \%$} \\
\hline Whole & $50.61 \pm 1.92$ & $48.67 \pm 1.96$ & $47.62 \pm 1.83$ & $46.08 \pm 3.41$ & $F=16.761$ & $<0.001^{*}$ \\
\hline Peripapillary & $53.83 \pm 2.67$ & $50.84 \pm 2.12$ & $50.47 \pm 1.89$ & $48.15 \pm 3.92$ & $F=19.204$ & $<0.001^{*}$ \\
\hline \multicolumn{7}{|c|}{ RNFL, median (IQR), $\mu \mathrm{m}$} \\
\hline Peripapillary & $116.00(110.50,120.75)$ & $113.00(102.00,121.00)$ & $109.00(101.00,127.50)$ & $115.00(96.00,137.00)$ & $H=1.154$ & $0.764^{\mathrm{a}}$ \\
\hline
\end{tabular}

RNFL, retinal nerve fibre layer; RPC, radial peripapillary capillary. * Statistically significant analysis of variance. ${ }^{\text {a }}$ Spearman correlation coefficient test.

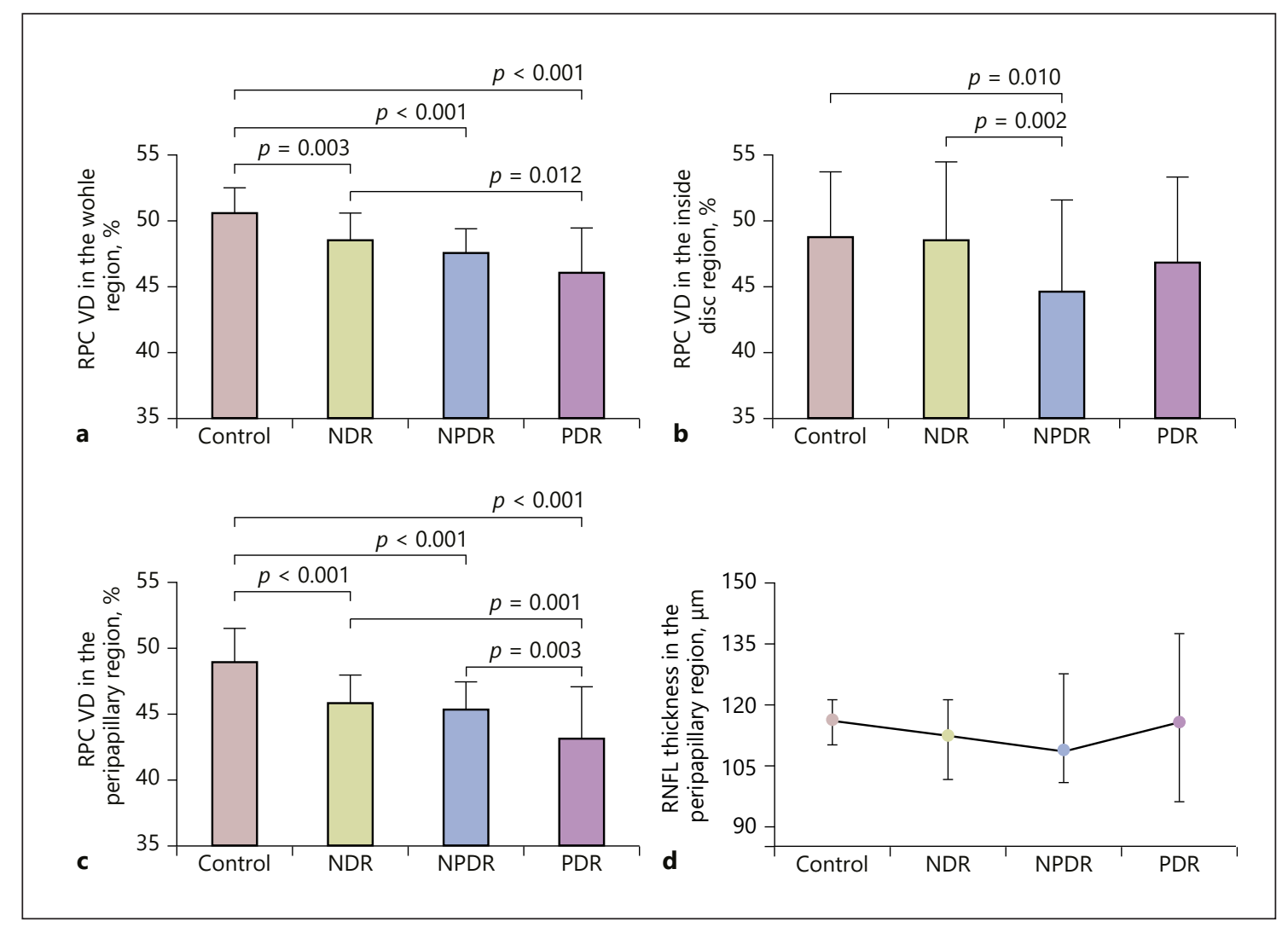

Fig. 2. Column of VD and RNFL thickness in the optic disc of each group (control, NDR, NPDR, and PDR). The means (bar) and standard deviations (whiskers) are illustrated in Table 2. Significant tests $(p<0.05)$ are marked with brackets in the figures. VD, vessel density; RNFL, retinal nerve fibre layer; RPC, radial peripapillary capillary.

inside disc, and peripapillary region and the pRNFL thickness of 4 groups are reported in Table 2 and Figure 2. The one-way analysis of variance showed that the average RPC vessel density in the whole region of the NDR $(48.67 \% \pm$ $1.96 \%)$, NPDR $(47.62 \% \pm 1.83 \%)$, and PDR $(46.08 \% \pm$ $3.41 \%)$ groups was significantly lower than that of the control group $(50.61 \% \pm 1.92 \%, p<0.001)$. The average
RPC vessel densities of the inside disc region were $48.88 \%$ $\pm 4.92 \%, 49.87 \% \pm 4.53 \%, 44.79 \% \pm 6.85 \%$, and $46.92 \% \pm$ $6.40 \%$ in the control, NDR, NPDR, and PDR groups, respectively, and these values were significantly different $(p=0.009)$. The average RPC vessel density in the peripapillary region of the NDR $(50.84 \% \pm 2.12 \%)$, NPDR $(50.47 \%$ $\pm 1.89 \%)$, and PDR $(48.15 \% \pm 3.92 \%)$ groups was also sig- 
nificantly lower than those of the control group (53.83\% $\pm 2.67 \%, p<0.001)$. The Kruskal-Wallis test showed that the pRNFL thickness had no significant difference amongst the groups ( $p=0.764$, Table 2$)$. However, the pRNFL thickness showed a trend of decreasing first and then increasing (Fig. 2d). On a subgroup analysis using a multiple-comparison test based on the stage of the $\mathrm{DR}$, the average RPC vessel density in the whole region between the control and the NDR $(p=0.003)$, the control and the NPDR $(p<0.001)$, the control and the PDR $(p<0.001)$, and the NDR and the PDR $(p=0.012)$ groups showed significant differences (Fig. 2a), and the average RPC vessel density in the peripapillary region between the control and the NDR $(p<0.001)$, the control and the NPDR ( $p<$ $0.001)$, the control and the PDR $(p<0.001)$, the NDR and the PDR $(p=0.001)$, and the NPDR and the PDR ( $p=$ 0.003 ) showed significant differences, whereas the other comparisons showed no significant difference (Fig. 2c). The inside disc RPC vessel density in the NPDR group was significantly decreased compared with those in the control $(p=0.01)$ and the NDR $(p=0.002)$ groups (Fig. $2 b)$. Table 3 displays the peripapillary region divided into 8 sections. Compared with the control group, the PDR, NPDR, and NDR groups had significantly reduced vessel density in 8, 5 (i.e., NS, NI, IN, IT, and TS), and 4 (i.e., NS, NI, IN, and IT) subregions, respectively (all $p<0.05$ ). The pRNFL thickness of the NDR group was significantly reduced in the IN subregion compared with that of the control group $(p=0.001)$, whereas the pRNFL thickness of the PDR group was significantly increased in the TS and the TI subregions compared with that of the control group (all $p<0.01)$.

\section{Correlations amongst RPC Vessel Density, pRNFL}

\section{Thickness, and DR Severity}

The average RPC vessel density in the whole $(r=$ $-0.590, p<0.001)$ and the peripapillary $(r=-0.583, p<$ 0.001 ) regions was significantly correlated with DM severity. However, no statistically significant association was found between the inside disc RPC vessel density and the DR severity $(r=-0.191, p=0.050)$ and between the pRNFL thickness and the DR severity $(r=-0.045, p=$ 0.648 ; Table 4 ). The results of Spearman correlation analysis between the RPC vessel density and the pRNFL thickness in the peripapillary region amongst different groups are shown in Figure 3. The correlation between the peripapillary RPC vascular density and the pRNFL thickness in the PDR group was not statistically significant $(r=$ $0.394, p=0.05$ ), whereas that in the other groups was positively correlated.

Analysis of Neurovascular Changes in

Diabetics' Optic Disc Using OCTA

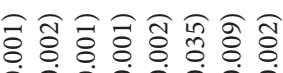

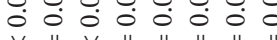

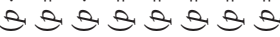

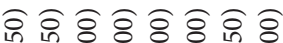
भ क in in in in in in

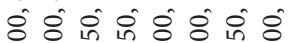

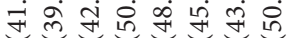
88888888

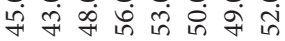

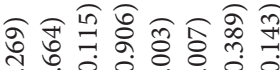
กิ $\theta, 3, a, 3, a$

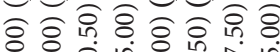

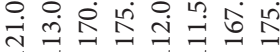
ถิ \&ิ กั่ \&8 8 8 8 8 +i

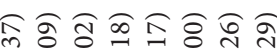

\% II II II II II ||

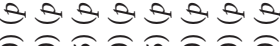

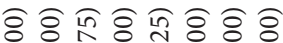
in 辛 in in in in in in ผ

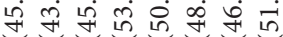

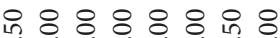
भ के

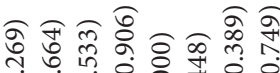
000 . s $s$ s

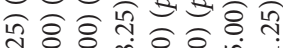

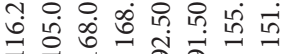

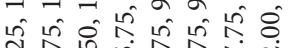
बूँ 요 8 8 8 요 8 in 8

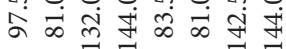

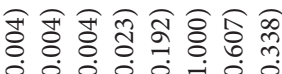
0 00000100 $\Leftrightarrow, \Leftrightarrow, s, a, s$

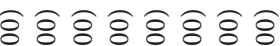
完条

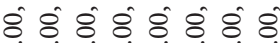

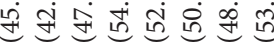
ঃ: : ৪ : ৪ :

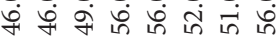

ลิธิ) กิ

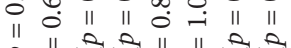

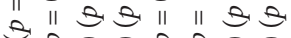
औ 은 응 응 항 के मे 연

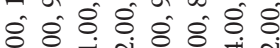
ت্ৰ ৪ : 8: ৪ : i

ฮิณ กิ

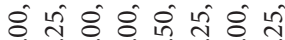

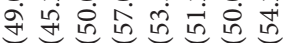
8 응 \& $8 \circ \circ$ जा ने हिं

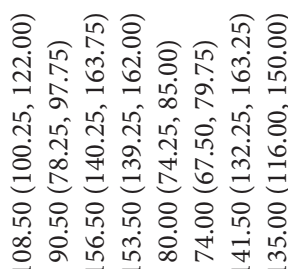

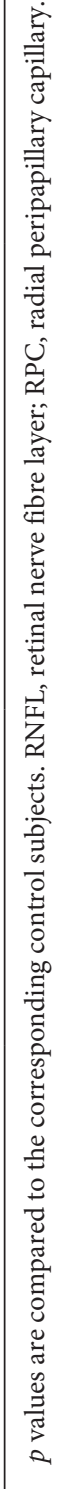

Ophthalmic Res 2021;64:951-959 DOI: 10.1159/000517145 


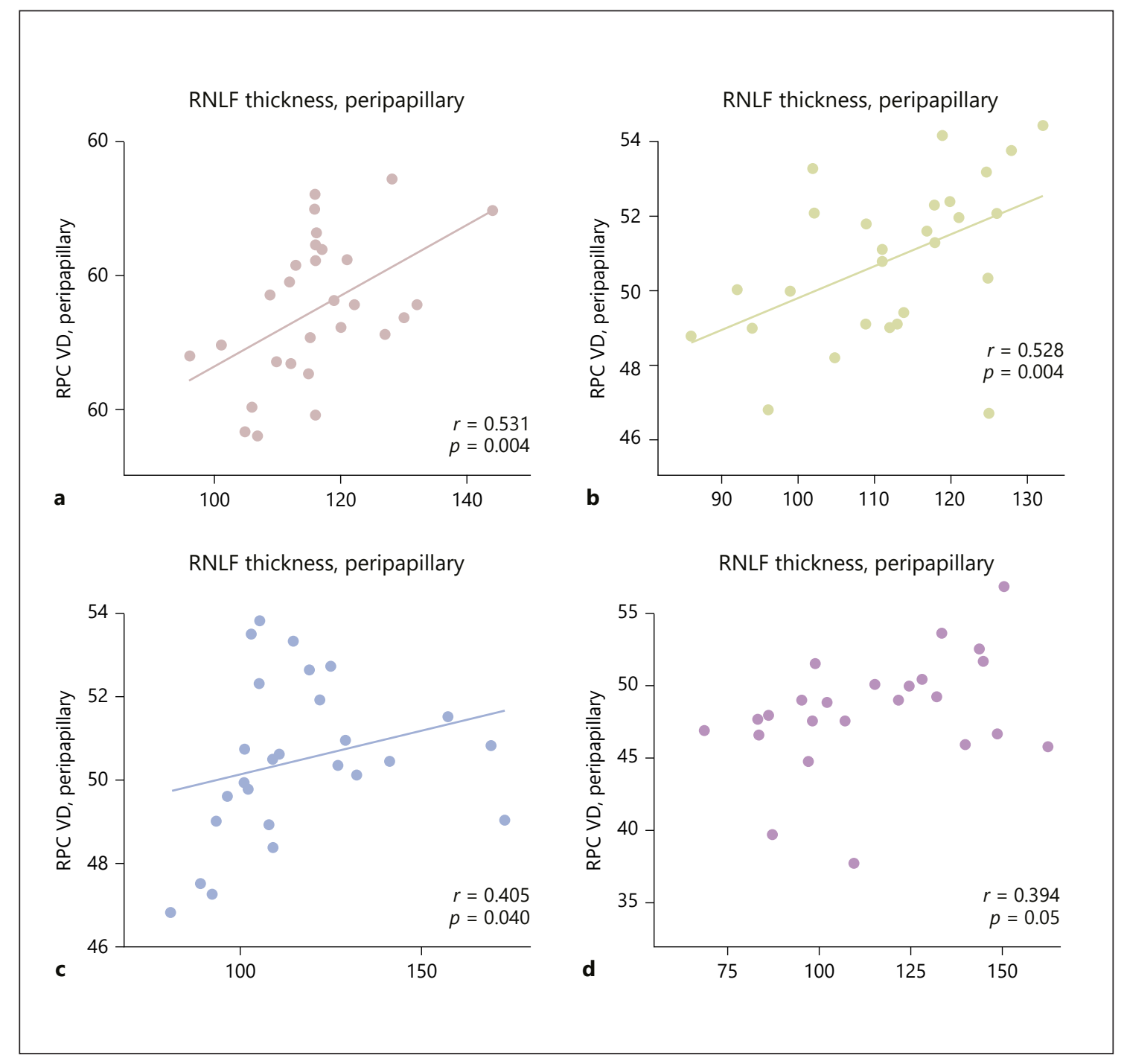

Fig. 3. Scatter plots of the linear relationships between RPC VD and RNFL thickness of the peripapillary region in the control (a), NDR (b), NPDR (c), and PDR (d) groups. VD, vessel density; RNFL, retinal nerve fibre layer; $\mathrm{RPC}$, radial peripapillary capillary.

Table 4. The relationship between RPC vessel density or pRNFL thickness and DR severity

\begin{tabular}{llrll}
\hline \multirow{2}{*}{$\begin{array}{l}\text { DR } \\
\text { severity }\end{array}$} & \multicolumn{2}{l}{ RPC vessel density } & $\begin{array}{l}\text { pRNFL } \\
\text { thickness }\end{array}$ \\
\cline { 2 - 4 } & whole & inside disc & peripapillary & \\
\hline$r$ & -0.590 & -0.191 & -0.583 & -0.045 \\
$p$ value & $<0.001^{* *}$ & 0.050 & $<0.001^{* *}$ & 0.648 \\
\hline
\end{tabular}

Spearman's rank correlation coefficient was utilized to examine relationships. RNFL, retinal nerve fibre layer; RPC, radial peripapillary capillary; DR, diabetic retinopathy. ${ }^{* *} p<0.01$.

\section{Discussion}

This study reveals the changes in the RPC vessel density and the pRNFL thickness at different stages of DR. The quantitative method used in previous studies on DR has analysed the macular perfusion parameters, such as perfusion density, foveal avascular zone area, and ganglion cell complex thickness [19, 27-29]. Many studies have confirmed that the progression of DM is accompanied with the aggravation of macular ischaemia. However, few studies attempt to scan the optic disc. It has been shown that the peripapillary region has an earlier microvascular susceptibility than the macula [30]. Here, we aim 
to provide complete data and deep insight into the progressive changes in DR in the optic disc.

The central retinal artery is responsible for the blood supply of the RNFL, and the posterior ciliary artery and few branches of the central retinal artery supply the deeper layer of the optic disc, which is in the vicinity of the sieve plate area [31]. Slender RPC vessels are the superficial capillary network of RNFL, which easily form anastomosis with the surrounding blood vessels. Some researchers have found a positive correlation between RPC and RNFL in healthy persons, which is also found in our study [32, 33]. Vujosevic et al. [30] found a significant positive association between them not only in healthy individuals but also in diabetic patients without DR, which was also found in our study. Thus, RPC is closely linked to RNFL, and RPC may be responsible for RNFL nourishment $[22,34]$. Furthermore, we found no correlation between RPC and RNFL in patients with PDR, however possibly due to the pseudo-thickening of the nerve fibre layer caused by retinal oedema. Our study shows that compared with healthy subjects, the patients in the NDR, NPDR, and PDR groups have reduced RPC vessel density in 4,5 , and 8 peripapillary sectors. Therefore, microvascular changes may be an early and reliable marker for preclinical DR. This result is similar to that in previous studies. However, some inadequacies exist in their studies. For instance, Cao et al. [24] have not included the NPDR and the PDR groups, and Li et al. [25] have not incorporated the PDR group. Both researchers cannot determine the progressive variation in the OCTA parameters with DR aggravation. Our study has confirmed a negative correlation between peripapillary RPC and DR aggravation. DR is primarily characterized by capillary reorganization and degeneration [35]. Several mechanisms may explain the reduced RPC vessel density. First, hyperglycaemia leads to the destruction of the capillary structure, which is manifested by the thickening of the basement membrane and loss of pericytes, damaging the integrity of the vascular endothelium [36]. In turn, the leaked fluid presses the blood vessels to stenosis or even occlusion, resulting in vessel density reduction [37]. Second, retinal perfusion is affected by the reduced erythrocyte deformability and elevated blood viscosity in patients with DM [38]. In addition, the high energy requirements of RNFL's unmyelinated nerve fibres make RPC extremely sensitive to ischaemic injury [32]. Thus, poor $\mathrm{RPC}$ regulation may also be one of the reasons for such a situation [39].

Interestingly, we found there was no significant trend in vessel density of the inside disc region between the 4 groups. First, the vessel density of the NPDR group had a statistically significant reduction both in comparison with the control group and the NDR group, which is consistent with the mechanism of the RPC vessel density reduction mentioned above. Second, the comparisons between other groups were not found to be statistically different. We speculate that the trend of increasing RPC vessel density at the PDR stage may be due to the presence of slender neovascularization on the disc, which is difficult to detect and inevitably introduced errors; the increased trend of RPC vessel density during the NDR stage may be due to the more crowded anatomy of the peripapillary region compared with the neurovascular distribution in other regions of the retina, which makes it easier to generate compensatory mechanism due to ischaemia. These speculations, however, await further validation.

Furthermore, the average pRNFL thickness tends to decrease in patients with NDR and NPDR. These results are consistent with the recently published studies $[25,40]$. Through 8 partitions of pRNFL, we have found that the pRNFL thickness in NDR is considerably decreased only in inferior nasal sectors than that in healthy subjects. Differences in the peripapillary RPC vessel density are also concentrated in the inferior and the nasal regions, which may explain the trophic effect of RPC on RNFL [32]. Satue et al. [21] believe that the main difference in pRNFL thickness between the patients without DR and the healthy subjects is concentrated in the inferior quadrant, and Shin et al. [17] have confirmed that the neurodegenerative changes caused by DM primarily affect the inferior quadrant of pRNFL. Vujosevic et al. [30] found an early reduction of RNFL in the inferior quadrant even in diabetic patients without DR. Their studies have great similarity with our study. However, these studies use optical coherence tomography to measure the thickness of pRNFL. Differences in partitioning may be the reason for the nuances.

Notably, our study has found no correlation between pRNFL thickness and the aggravation of DR. This finding is consistent with the recently published study [40]. The reasonable explanation for this manifestation is that the RPC perfusion reduction at the early stage results in $\mathrm{pRN}$ FL nutritional impairment and progressive loss of ganglion and glial cells, and fluid leakage and tissue oedema at the late stage lead to pRNFL pseudo-thickening [41]. This phenomenon is also the reason for the absence of correlation between the peripapillary RPC vessel density and the pRNFL thickness in patients with PDR, but negative correlations are found between patients with DM at varying diabetic stages and healthy subjects. Thus, judg- 
ing the severity of DR by evaluating pRNFL thickness may not be advisable.

Interestingly, diabetics at any stage show significant changes in most areas of the perinipple RPC than in those in pRNFL compared with the healthy subjects. This finding suggests that OCTA microvascular changes are more sensitive and extensive than neural structural changes and that the changes in the RPC vessel density and the pRNFL thickness do not occur simultaneously, but the cause and effect relationship between them is uncertain.

Limitations exist in this study. First, the OCTA selects a relatively small scan range. Research shows that the RPC in the arcuate fibre regions still exists in the $5.5-\mathrm{mm}$ range, which extends outward from the optic disc [22, 42]. However, the clarity of the blood flow signal decreases with increasing scanning range. Second, further studies based on longitudinal data rather than cross-sectional data should be made, which can be used to investigate the causal relationship between the reduced peripapillary perfusion and the reduced pRNFL thickness. Third, whether DM medication therapy affects retinal parameters is unknown, which may be one of the reasons for the different conclusions. Last, we have ruled out patients with diabetic macular oedema, which affects the comprehensive understanding of the neurovascular changes in the optic disc of DR. However, with these criteria, we have excluded the factors affecting pRNFL thickness due to macular oedema.

In general, we think that the vascular indicator of the peripapillary RPC perfusion decreases with the aggravation of the disease, which occurs in preclinical DR, but the sensitivity of the pRNFL thickness as a morphological indicator is lower than that as a vascular indicator. Currently, the treatment of DR is limited, applies principally to the advanced stage, and has short-term efficacy and side effects [43]. Early screening should be the mainstay of DR to achieve awareness of the preclinical disease and delay the progression. As a non-invasive and rapid detection method, the OCTA can quantify blood perfusion and morphological changes in the retina of patients with preclinical DR.

\section{Statement of Ethics}

This protocol was guided by the Declaration of Helsinki and was approved by the Research Ethics Committee of the Affiliated Hospital of Weifang Medical University (Approval No. 2019-010). Informed, written consent was obtained from all participants.

\section{Conflict of Interest Statement}

The authors have no conflicts of interest to declare.

\section{Funding Sources}

No funding was available to support this article.

\section{Author Contributions}

Fan Jia designed experiments; Yan Li and Na Li directed, reviewed, and checked the experiments; Fan Jia, Min Zhang, Chunyuan Song, and Jin Yang carried out experiments; Fan Jia and Min Zhang analysed experimental results; Min Zhang wrote the manuscript and drew the statistical charts; Yan Li and Shuna Wang modified the language; Shuna Wang, Kaili Yang, and $\mathrm{Na} \mathrm{Li}$ assisted in data collection.

\section{Data Availability Statement}

All data generated or analysed during this study are included in this published article.

\section{References}

1 Saeedi P, Petersohn I, Salpea P, Malanda B, Karuranga S, Unwin N, et al. Global and regional diabetes prevalence estimates for 2019 and projections for 2030 and 2045: results from the International Diabetes Federation diabetes atlas, 9(th) edition. Diabetes Res Clin Pract. 2019 Nov; 157:107843.

2 Standl E, Khunti K, Hansen TB, Schnell O. The global epidemics of diabetes in the 21st century: current situation and perspectives. Eur J Prev Cardiol. 2019 Dec;26(2_suppl):7-14.

3 Wong TY, Cheung CM, Larsen M, Sharma S, Simó R. Diabetic retinopathy. Nat Rev Dis Primers. 2016 Mar 17;2:16012.
4 Yau JW, Rogers SL, Kawasaki R, Lamoureux EL, Kowalski JW, Bek T, et al. Global prevalence and major risk factors of diabetic retinopathy. Diabetes Care. 2012 Mar;35(3):556-64.

5 Lee R, Wong TY, Sabanayagam C. Epidemiology of diabetic retinopathy, diabetic macular edema and related vision loss. Eye Vis. 2015 Sep 30;2:17.

6 Wang W, Lo ACY. Diabetic retinopathy: pathophysiology and treatments. Int J Mol Sci. 2018 Jun 20;19(6):1816.

7 Beltramo E, Porta M. Pericyte loss in diabetic retinopathy: mechanisms and consequences. Curr Med Chem. 2013;20(26):3218-25.
8 Tien T, Zhang J, Muto T, Kim D, Sarthy VP, Roy S. High glucose induces mitochondrial dysfunction in retinal müller cells: implications for diabetic retinopathy. Invest Ophthalmol Vis Sci. 2017 Jun 1;58(7): 2915-21.

9 Sasaki M, Ozawa Y, Kurihara T, Kubota S, Yuki K, Noda K, et al. Neurodegenerative influence of oxidative stress in the retina of a murine model of diabetes. Diabetologia. 2010 May;53(5):971-9.

10 Lynch SK, Abràmoff MD. Diabetic retinopathy is a neurodegenerative disorder. Vision Res. 2017 Oct;139:101-7. 
11 Antonetti DA, Barber AJ, Bronson SK, Freeman WM, Gardner TW, Jefferson LS, et al. Diabetic retinopathy: seeing beyond glucoseinduced microvascular disease. Diabetes. 2006 Sep;55(9):2401-11.

12 Song P, Yu J, Chan KY, Theodoratou E, Rudan I. Prevalence, risk factors and burden of diabetic retinopathy in China: a systematic review and meta-analysis. J Glob Health. 2018 Jun;8(1):010803.

13 Perkins BA, Olaleye D, Zinman B, Bril V. Simple screening tests for peripheral neuropathy in the diabetes clinic. Diabetes Care. $2001 \mathrm{Feb}$;24(2):250-6.

14 Barber AJ, Lieth E, Khin SA, Antonetti DA, Buchanan AG, Gardner TW. Neural apoptosis in the retina during experimental and human diabetes. Early onset and effect of insulin. J Clin Invest. 1998 Aug 15;102(4):783-91.

15 Kwiterovich KA, Maguire MG, Murphy RP, Schachat AP, Bressler NM, Bressler SB, et al. Frequency of adverse systemic reactions after fluorescein angiography. Results of a prospective study. Ophthalmology. 1991 Jul;98(7): 1139-42.

16 Yannuzzi LA, Rohrer KT, Tindel LJ, Sobel RS, Costanza MA, Shields W, et al. Fluorescein angiography complication survey. Ophthalmology. 1986 May;93(5):611-7.

17 Shin YI, Nam KY, Lee SE, Lee MW, Lim HB, Jo YJ, et al. Peripapillary microvasculature in patients with diabetes mellitus: an optical coherence tomography angiography study. Sci Rep. 2019 Nov 1;9(1):15814.

18 Li Z, Alzogool M, Xiao J, Zhang S, Zeng P, Lan Y. Optical coherence tomography angiography findings of neurovascular changes in type 2 diabetes mellitus patients without clinical diabetic retinopathy. Acta Diabetol. 2018 Oct; 55(10):1075-82.

19 Yang D, Cao D, Huang Z, Xie J, Meng Q, Dong $X$, et al. Macular capillary perfusion in Chinese patients with diabetic retinopathy obtained with optical coherence tomography angiography. Ophthalmic Surg Lasers Imaging Retina. 2019 Apr 1;50(4):e88-95.

20 Kim K, Kim ES, Kim DG, Yu SY. Progressive retinal neurodegeneration and microvascular change in diabetic retinopathy: longitudinal study using OCT angiography. Acta Diabetol. 2019 Dec;56(12):1275-82.

21 Satue M, Cipres M, Melchor I, Gil-Arribas L, Vilades E, Garcia-Martin E. Ability of swept source OCT technology to detect neurodegeneration in patients with type 2 diabetes mellitus without diabetic retinopathy. Jpn J Ophthalmol. 2020 Jul;64(4):367-77.
22 Yu PK, Cringle SJ, Yu DY. Correlation between the radial peripapillary capillaries and the retinal nerve fibre layer in the normal human retina. Exp Eye Res. 2014 Dec;129:83-92.

23 Yarmohammadi A, Zangwill LM, Diniz-Filho A, Suh MH, Yousefi S, Saunders LJ, et al. Relationship between optical coherence tomography angiography vessel density and severity of visual field loss in glaucoma. Ophthalmology. 2016 Dec;123(12):2498-508.

24 Cao D, Yang D, Yu H, Xie J, Zeng Y, Wang J, et al. Optic nerve head perfusion changes preceding peripapillary retinal nerve fibre layer thinning in preclinical diabetic retinopathy. Clin Exp Ophthalmol. 2019 Mar;47(2):21925.

25 Li Z, Wen X, Zeng P, Liao Y, Fan S, Zhang Y, et al. Do microvascular changes occur preceding neural impairment in early-stage diabetic retinopathy? Evidence based on the optic nerve head using optical coherence tomography angiography. Acta Diabetol. 2019 May; 56(5):531-9.

26 Wilkinson CP, Ferris FL 3rd, Klein RE, Lee PP, Agardh CD, Davis M, et al. Global Diabetic Retinopathy Project Group. Proposed international clinical diabetic retinopathy and diabetic macular edema disease severity scales. Ophthalmology. 2003 Sep;110(9): 1677-82.

27 Vujosevic S, Toma C, Villani E, Gatti V, Brambilla M, Muraca A, et al. Early detection of microvascular changes in patients with diabetes mellitus without and with diabetic retinopathy: comparison between different swept-source OCT-A instruments. J Diabetes Res. 2019 Jun 122019;2019:2547216.

28 Motulsky EH, Liu G, Shi Y, Zheng F, Flynn HW Jr, Gregori G, et al. Widefield sweptsource optical coherence tomography angiography of proliferative diabetic retinopathy. Ophthalmic Surg Lasers Imaging Retina. 2019 Aug 1;50(8):474-84.

29 Jo YH, Sung KR, Shin JW. Effects of age on peripapillary and macular vessel density determined using optical coherence tomography angiography in healthy eyes. Invest Ophthalmol Vis Sci. 2019 Aug 1;60(10):3492-8.

30 Vujosevic S, Muraca A, Gatti V, Masoero L, Brambilla M, Cannillo B, et al. Peripapillary microvascular and neural changes in diabetes mellitus: an OCT-angiography study. Invest Ophthalmol Vis Sci. 2018 Oct 1;59(12):507481.

31 Hayreh SS. Blood supply of the optic nerve head. Ophthalmologica. 1996;210(5):285-95.

32 Mansoori T, Sivaswamy J, Gamalapati JS, Balakrishna N. Topography and correlation of radial peripapillary capillary density network with retinal nerve fibre layer thickness. Int Ophthalmol. 2018 Jun;38(3):967-74.
33 Falavarjani KG, Shenazandi H, Naseri D, Anvari $P$, Sedaghat A, Hashemi M, et al. Correlation of optic disc morphometry and optic disc microvasculature assessed with optical coherence tomography angiography. Can J Ophthalmol. 2018 Dec;53(6):595-9.

34 Chan G, Balaratnasingam C, Xu J, Mammo Z, Han S, Mackenzie P, et al. In vivo optical imaging of human retinal capillary networks using speckle variance optical coherence tomography with quantitative clinico-histological correlation. Microvasc Res. 2015 Jul;100: 32-9.

35 Tooke JE. Microvascular haemodynamics in diabetes. Eye. 1993; 7(Pt 2):227-9.

36 Li T, Jia Y, Wang S, Wang A, Gao L, Yang C, et al. Retinal microvascular abnormalities in children with type 1 diabetes mellitus without visual impairment or diabetic retinopathy. Invest Ophthalmol Vis Sci. 2019 Mar 1;60(4): 990-8.

37 Fujioka S, Karashima K, Nishikawa N, Saito Y. Correlation between higher blood flow velocity in the central retinal vein than in the central retinal artery and severity of nonproliferative diabetic retinopathy. Jpn J Ophthalmol. 2006 Jul-Aug;50(4):312-7.

38 Chang HY, Li X, Karniadakis GE. Modeling of biomechanics and biorheology of red blood cells in type 2 diabetes mellitus. Biophys J. 2017 Jul 25;113(2):481-90.

39 Hua D, Xu Y, Zeng X, Yang N, Jiang M, Zhang $\mathrm{X}$, et al. Use of optical coherence tomography angiography for assessment of microvascular changes in the macula and optic nerve head in hypertensive patients without hypertensive retinopathy. Microvasc Res. 2020 May; 129: 103969.

40 Liu L, Wang Y, Liu HX, Gao J. Peripapillary region perfusion and retinal nerve fiber layer thickness abnormalities in diabetic retinopathy assessed by OCT angiography. Transl Vis Sci Technol. 2019 Aug 1;8(4):14.

41 Chen X, Nie C, Gong Y, Zhang Y, Jin X, Wei $S$, et al. Peripapillary retinal nerve fiber layer changes in preclinical diabetic retinopathy: a meta-analysis. PLoS One. 2015 May 12;10(5): e0125919.

42 Jia Y, Simonett JM, Wang J, Hua X, Liu L, Hwang TS, et al. Wide-field OCT angiography investigation of the relationship between radial peripapillary capillary plexus density and nerve fiber layer thickness. Invest Ophthalmol Vis Sci. 2017 Oct 1;58(12):5188-94.

43 Rossino MG, Casini G. Nutraceuticals for the treatment of diabetic retinopathy. Nutrients. 2019 Apr 2;11(4):771.
Analysis of Neurovascular Changes in Diabetics' Optic Disc Using OCTA
Ophthalmic Res 2021;64:951-959

DOI: $10.1159 / 000517145$ 